\title{
Polymeric and Ceramic Nanoparticles in Biomedical Applications
}

\author{
Aura-Ileana Moreno-Vega, ${ }^{1}$ Teresa Gómez-Quintero, ${ }^{1}$ Rosa-Elvira Nuñez-Anita, ${ }^{2}$ \\ Laura-Susana Acosta-Torres, ${ }^{3}$ and Víctor Castaño ${ }^{4}$ \\ ${ }^{1}$ Licenciatura en Tecnología, Centro de Física Aplicada y Tecnología Avanzada, Universidad Nacional Autónoma de México, \\ Campus Juriquilla, Juriquilla, QRO, Mexico \\ ${ }^{2}$ Facultad de Medicina Veterinaria y Zootecnia, Universidad Michoacana de San Nicolás de Hidalgo, 58893 Morelia, MICH, Mexico \\ ${ }^{3}$ Unidad León, Escuela Nacional de Estudios Superiores, Universidad Nacional Autónoma de Mexico, León, GTO, Mexico \\ ${ }^{4}$ Departamento de Ingeniería Molecular de Materiales, Centro de Física Aplicada y Tecnología Avanzada, Universidad Nacional \\ Autónoma de México, Campus Juriquilla, Boulevard Juriquilla 3001, 76230 Juriquilla, QRO, Mexico
}

Correspondence should be addressed to Víctor Castaño, meneses@unam.mx

Received 4 October 2012; Accepted 2 December 2012

Academic Editor: Mallikarjuna Nadagouda

Copyright ( $) 2012$ Aura-Ileana Moreno-Vega et al. This is an open access article distributed under the Creative Commons Attribution License, which permits unrestricted use, distribution, and reproduction in any medium, provided the original work is properly cited.

\begin{abstract}
Materials in the nanometer size range may possess unique and beneficial properties, which are very useful for different medical applications including stomatology, pharmacy, and implantology tissue engineering. The application of nanotechnology to medicine, known as nanomedicine, concerns the use of precisely engineered materials at this length scale to develop novel therapeutic and diagnostic modalities. Nanomaterials have unique physicochemical properties, such as small size, large surface area to mass ratio, and high reactivity, which are different from bulk materials of the same composition. Polymeric and ceramic nanoparticles have been extensively studied as particulate carriers in the pharmaceutical and medical fields, because they show promise as drug delivery systems as a result of their controlled- and sustained-release properties, subcellular size, and biocompatibility with tissue and cells. These properties can be used to overcome some of the limitations found in traditional therapeutic and diagnostic agents. Nanotechnology is showing promising developments in many areas and may benefit our health and welfare. However, a wide range of ethical issues has been raised by this innovative science. Many authorities believe that these advancements could lead to irreversible disasters if not limited by ethical guidelines.
\end{abstract}

\section{Introduction}

Recently, nanoparticles have been widely used in biomedical applications due to their specific physical and chemical properties which alter the normal biological activity, as compared to bulk materials [1]. The rise in the use of nanoparticles in this field therefore raises concern over the impact on human health such particles may have. This then requires the establishment of new regulations or adaptation of previous ones, based on a new definition of what needs to be regulated [2]. Such a science-based definition must be developed by several national and international standardization bodies, as well as organizations and authorities in order to have a definition that is broadly applicable to regulatory legislations.

\section{Nanoparticles Assessments}

2.1. A Working Definition of Nanoparticles. Based on this, European and other International Committees have defined a nanoparticle as a discrete entity which has three dimensions in the order of $100 \mathrm{~nm}$ or less. Nevertheless it is important to remember that the nanoscale $(1-100 \mathrm{~nm})$ used to describe nanoparticles should not be considered as strictly due to the variations that may exist during the nanoparticle measurement as well as the appearance of nanoscale properties in particles slightly above or below the nanoscale limits. This can include other important properties to take into account such as shape, surface area to mass ratio, and composition [2]. 
2.2. Size and Morphology Implications. Like everything else, the use of nanoparticles in biological systems has several aspects, both positive and contradictory. Undoubtedly, the size and how these nanoparticles are synthesized significantly influences the ease with which they come into biological systems and interact with tissues or cells. There are many studies which show the close relationship between these two variables, size and shape, and analyze the ease with which such nanoparticles circulate or accumulate within a living, special sites of sedimentation, and the time it takes for nanoparticles to saturate the system and promote cell functional failures. Nanoparticles move freely within a cell and can therefore interact with proteins, lipids, and other components [3].

For pharmaceutical purposes the nanoparticles should have a size range between $2 \mathrm{~nm}$ to $1 \mu \mathrm{m}$, allowing them to enter the body in a considerably fast manner. The most common routes, oral and other mucous membranes, face nanoparticles with the immune system, which triggers different reactions depending on the host organism. Although these nanoparticles are ideal vehicles for drug delivery into the body, they often accumulate in sites other than those therapeutically intended. Studies show comparisons between size, morphology, and surface of nanoparticles and the interactions and host responses. Many times nanoparticles are encapsulated to ensure a greater dose at the site of release, but this does not tackle the fact that the nanoparticles also disperse throughout the body and can cause various functional damages. Designing a nanoparticle can therefore be a challenge since the nanoparticle must be safe, easy to administer, and yet, nontoxic. In addition, little is known about the effects of size and shape of nanoparticles on the immune system. In vitro experiments have shown that nanoparticles of large sizes $(200 \mathrm{~nm})$ can easily penetrate human mucus barriers, altering their structure. It is thus important to study the complex effects of the different physicochemical and biological properties of nanoparticles on the modulation of the immune response [4].

The therapy efficiency of drug delivery via nanoparticles is reduced by the fact that the administration of medicine reaches the target site in a limited manner. For example, polyethyleneimine nanoparticles were used in a study to determine the destination and penetration of such nanoparticles when transiting through the blood brain barrier, and it was shown that a fixed size of nanoparticles $(50 \mathrm{~nm})$ promoted penetration of brain endothelial cells. Nanoparticles with different surface modifications interact in different ways with the surface of the brain epithelial cells, and many respond to metabolic inhibitors and endocytosis pathways (different surfaces have different forms of internalization). Consequently, many tests required size and surface morphology for efficient transport of nanoparticles [5].

Generalizing, each group of nanoparticles with different sizes, shapes, surfaces, chemical composition, and biopersistence (time a nanoparticle remains within the body) will have different effects on the health of the living. Toxicological studies show that closely similar substances induce substantially different responses. Some groups will be safe, whilst a group with an apparent slight variation may present significant toxic effects. Some ordinary raw materials are poisonous or harmful. However, when these were used to obtain nanoparticles, was the solutions to many medical complications, with minimal adverse effects. In conclusion, it is important to regulate manufacturing, consumption, routes of administration, and so forth especially in light of new developments and gained knowledge [6,7].

\section{Choice of Nanoparticles in Biomedicine}

3.1. Polymeric Nanoparticles. Polymers are macromolecules composed of a large number of repeating units organized in a chain-like molecular architecture exhibiting a multiplicity of compositions, structures, and properties. It is because of this variety of compositions, structures, and properties that polymers are being used in nanoparticle systems to generate nanoparticles suited for each specific biomedical application. The main use of polymeric nanoparticles is in drug delivery, although they are also used in bioimaging and biosensing assays [8].

The use of nanoparticles in drug delivery has received a lot of attention due to the emerging importance of targeted delivery in medicine, meaning that large amounts of research studies focused on generating polymeric nanoparticles that are efficient, tissue specific, and most importantly, nontoxic. For the preparation of nanoparticles for drug delivery, there are a variety of methods depending on how the drug will be loaded onto the nanoparticle. The resulting nanoparticle-drug compounds may have the structure of capsules, (polymeric nanoparticles or polymeric nanoconjugates), amphiphilic core/shell (polymeric micelles), or hyperbranched macromolecules of nanometer dimensions (dendrimers) [9].

3.2. Natural and Synthetic Polymeric Nanoparticles. Natural polymers such as chitosan, albumin, and heparin have been used for the delivery of oligonucleotides, DNA, and protein, as well as drugs. An albumin-paclitaxel nanoconjugate has been studied in the treatment of metastatic breast cancer during phase III clinical trials [10]. Furthermore, natural polymers and synthetic polymers for nanoparticles such as $\mathrm{N}$-(2-hydroxypropyl)-methacrylamide, copolymer (HPMA), poly(ethylene glycol) (PEG), poly(lactic acidglycolic acid) (PLGA), and poly(lactic acid) PLA are used. Different in vitro and in vivo research studies have focused on the use of conjugated polymeric nanoparticles with chemotherapeutic drugs to reduce the damaging effects of the free drug administration [9]. Figure 1 shows film formation of PLGA.

For both natural and synthetic polymers, polymeric nanoparticle drug delivery systems allow the particle to be more target specific since the coating of the nanoparticles with polymers increases the amount of drug-loaded as well as tissue/cell-specific recognition proteins, which generates a more targeted and efficient nanoparticle. Examples of such nanoparticle systems in cancer treatment are ternary structures composed of a ligand or an antibody (targeting moiety), a polymer which acts as the carrier, and 


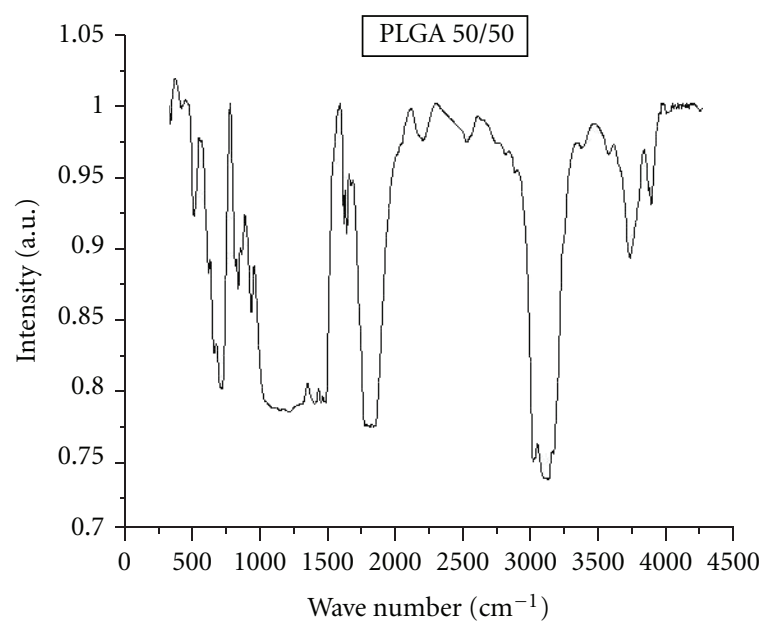

(a)

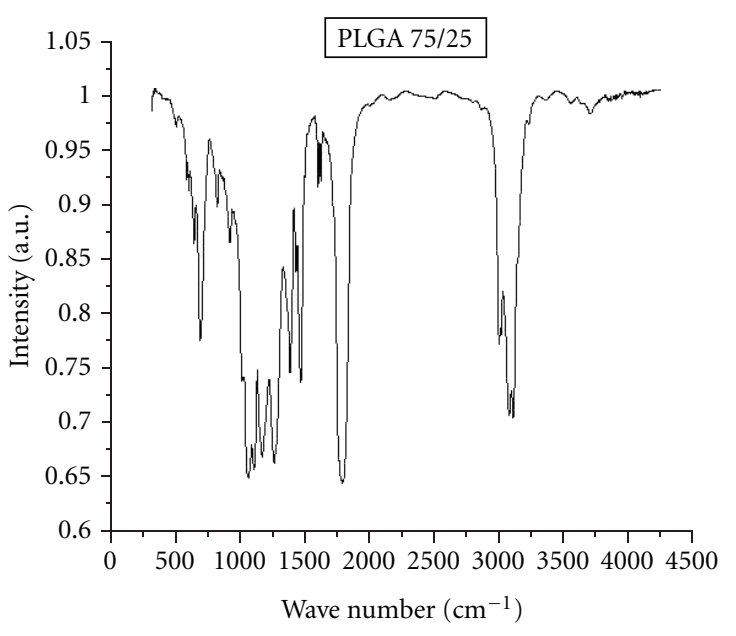

(b)

FIgURE 1: Infrarred spectra of PLGA 50/50 and PLGA 75/25 crystals, when dissolved in chloroform.

an active chemotherapeutic drug [9]. Table 1 presents different polymer nanoparticles acting as nanocarriers in biomedical fields.

3.3. Polymeric Micelles. Micelles are biocompatible nanoparticles varying in size from 50 to $200 \mathrm{~nm}$ in which poorly soluble drugs can be encapsulated. Polymers are used in core-shell nanoparticles because they offer a wide range of applications from drug delivery to bioimaging. A polymeric core-shell nanostructure comprises a polymeric core and/or a polymeric shell and can be dispersed in a matrix of any material class whose property is to be modified or enhanced. In drug delivery, polymeric-based micellar systems which contain a hydrophobic core surrounded by hydrophilic polymers are used as carriers for hydrophobic drugs. These systems offer various advantages including easy preparation, efficient drug loading, and controlled release kinetics. Various cancer-related drugs such as paclitaxel, doxorubicin, 5fluoracil, 9-nitrocamptothecin, cisplatin, triptorelin, dexamethasone, and xanthone, have been successfully encapsulated on PLGA, PLA and PCL nanoparticles [11]. Growing evidence is taken in account for nano/microparticle-based delivery system for macromolecular therapeutics. Biodegradable nano/microparticles of poly(D,L-Lactate-co-glycolide) (PGLA) and PLGA-based polymers are explorer as carriers for controlled delivery macromolecules such as proteins, peptides, vaccines, genes, antigens, and growth factors. A major difficult to this technology is about drug encapsulation [12]. More research is in progress to address such challenging problems. On the other hand, multifunctional coreshell nanospheres made of amphiphilic co-polymer micelles whith core bio-functionalized $\mathrm{Au}$ or CdSe nanoparticles (hydrophobic block) are protected with a PEG coating (hydrophilic block) which provides nanoscopic sensing and slow-targeted drug release [13].

Another great advantage of polymeric micelles is the synthesis of $\mathrm{pH}$-sensitive drug delivery systems which can be engineered to release their contents or change their physicochemical properties in response to variations in the acidity or surroundings. Other authors prepared and characterized polymeric micelles composed of amphiphilic pH-responsive poly(N-isopropylacrylamide) (PNIPAM) or poly(alkyl(meth)acrylate) derivatives [14]. Acidification of the PNIPAM copolymers induces a coil-to-globule transition that can be exploited to destabilize the intracellular vesicle membranes. Furthermore, poly(alkyl(meth)acrylate) copolymers can be designed to interact with either hydrophobic drugs or polyions and release their cargo upon an increase in $\mathrm{pH}$. Recently, 20-45 nm polymeric-based micelles has been investigated, which comprise a hydrophobic PLLA core and a hydrophilic PEG shell conjugated to TAT, a highly $\mathrm{pH}$-sensitive and cell penetrating polymer. It has been shown that TAT micelles seem attractive for targeting acidic solid tumours [15].

As drug carriers and releasers, polymeric micelles may be used to deliver a drug passively or actively although it may also be attached to a surface, to create a controlled, drug releasing surface with various applications in tissue engineering and body implants. Growth and differentiation polypeptides such as epidermal growth factor, basic fibroblast growth factor, and members of the transforming growth factor beta family have been covalently immobilized to surfaces of polymeric materials, particles and structures for tissue engineering, and regenerative medicine applications. In a NZW rabbit model of long bone distraction osteogenesis, it was shown that a single injection of rhBMP7-loaded polymeric core shell nanoparticles accelerated new bone regeneration and consolidation [13].

Finally, polymeric micelles are also obtaining attention in the field of biosensing, since enzymes may also be adsorbed onto the surfaces of polymeric nanoparticles, generating more precise biosensing systems. Moreover, functionalized PEGylated gold nanoparticles have been developed that can be reversibly associated through the addition of lectin, producing an accompanying color change [16]. Due to the high stability and nonfouling characteristics of these 


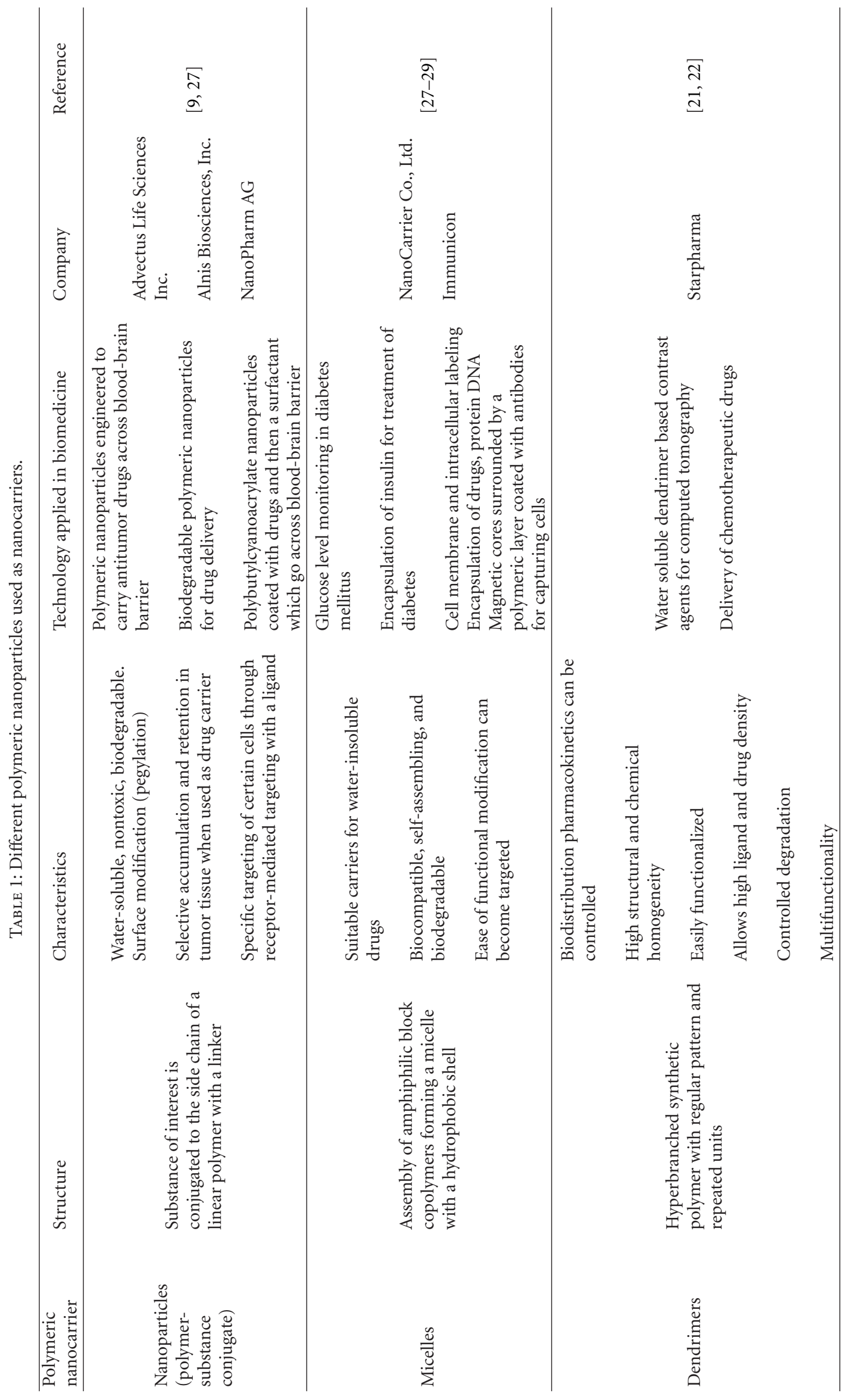


nanoparticles, they may be useful for diagnostics in bloodserum-containing samples. Another interesting application of polymeric nanoparticles for biosensing systems involves immobilized enzymes on core-shell polymeric nanoparticles or catalysts encapsulated suitably into nanoparticles, to elevate their activity [17].

3.4. Biodegradable Polymers. Some other main group is the biodegradable polymeric nanoparticles because of the important contribution as drug delivery systems. Currently, there are only a small number of commercially available products that utilize this technology. Biodegradable NPs have been used for the control release of several drugs like vaccines, prepared by the spontaneous emulsification/solvent human growth hormone, insulin, antitumor agents, and also contraceptives. Long circulation of rate and the impact of drug/polymer ratio on the size drugs in the body is the key in the successful drug of NPs [18].

It has been discussed about biodegradable polymeric NPs with appropriated surface modification that can deliver drugs to diagnostic and therapeutic applications in neurological disorders such as Alzheimer's disease (AD). Polymeric NPs are the promising candidates to deliver drugs in central nervous system. However, the challenges ahead to resolve the question of binding of the drugs (loaded onto nanoparticles) to amyloid plaques that are used as target for developing the biological markers [19]. The therapeutics advancement of neurodegenerative disorders such as $\mathrm{AD}$ is difficult due to restrictive mechanism to cross the blood brain barrier (BBB). Nowadays, NPs radiolabeled and tissue specific [(125 IClioquinol) (CQ, 5-cloro-7-iodo-8-hydroxyquinoleine-NP)] based on polymeric devices are developed to diagnosis of $\mathrm{AD}$ as the promising delivery vehicle for in vivo detection of amyloid plaques. In fact, it has been proposed that these could cross the BBB [20].

3.5. Dendrimers. Dendrimers are synthetic polymeric macromolecules of nanometer dimensions that composed of multiple highly branched monomers that emerge radially from the central core. Their structure offers various advantages such as monodisperse and controllable size, modifiable surface, functionality, multivalency, water solubility, and an available internal cavity for drug delivery [9]. The resultant spherical macromolecular structure has a size similar to albumin and hemoglobin, although it is smaller than multimers like the IgM antibody complex [21]. The characteristic arquitecture of dendrimers and the flexibility in the modification of their structure has allowed a greater progress in the application of biocompatible dendrimers for targeted drug delivery. Regarding this, there are studies on the use of biocompatible dendrimers for cancer treatment to deliver chemotherapeutic drugs such as cisplatin and doxorubicin [22].

3.6. Nanoparticle-Cell Interaction. Polymeric nanocarriers are easy to biofunctionalize with proteins, oligonucleotides, polysaccharides, or even DNA, allowing the nanoparticles to interact with the surrounding cells in the tissue they are targeted towards. Polymer coating of nanoparticles not only allows for biofunctionalization of the particles but also diminishes the clearing rate of such particles. Many nanoparticles have hydrophobic surfaces and are therefore rapidly opsonized and cleared by the bodily reticuloendothelial and mononuclear phagocytic systems becoming useless for targeted therapeutics which requires the persistence of the nanoparticulate systems throughout the systemic circulation. The coating and modification of surfaces with hydrophilic polymers therefore promote nanoparticle-cell interactions as well as internalization. Furthermore, the polymeric coatings (nanoshells) create a cloud of chains at the nanoparticle surface which repels plasma proteins. Examples of polymeric coatings include PEG (polyethylene glycol), PVO (poly-vinyl octanal acetal), or chitosan derivatives [11].

Substantial studies have been directed towards developing safe and efficient chitosan-based particles for drugs delivery systems. Chemically modified chitosan or its derivatives have been analyzed since past decade to evaluate the usefulness in delivering a variety of bioactive molecules [23]. The discovery of synthetic small interfering RNA (siRNA) technology for gene therapy has led to a surge of interest for developing siRNA-loaded nanocomplexes of chitosan and its derivatives for silencing genes. Efficient intracellular uptake requires suitable carriers due to that siRNA do not freely cross the cell membrane. In this sense, nonviral vectors such as chitosan or its derivatives are attractive, taking in to account these polymers are biodegradable, biocompatible, with low toxicity and high cationic potential [24]. It has been accepted that siRNA can be a powerful therapeutic drug, but its delivery remains a major challenge. Cyclodextrins (CDs), which are natural cyclic oligosaccharides, have been applied as delivery vehicles for siRNA, particularly, for the treatment of solids tumors; recently it has been demonstrated as clinical success [25]. Another example to useful polymeric devices such as cyclodextrins is related to oral insulin delivery which was developed to increase the residence time of insulin near the intestinal absorptive cells [26].

The use of polymers in the fabrication of nanoparticles has received a lot of attention due to the large variety of structures that may be obtained from a combination of properties of individual materials. So far, the majority of commercial polymeric-incorporated nanoparticle applications in medicine are focused towards bioactive, cost-effective, and controllable therapeutic agent delivery. However, as mentioned on some examples before, there are still many potential applications for polymeric nanoparticles such as bioimaging, biosensing, and antitumor therapies.

As with all nanoparticles, cytotoxicity and degradation by-products remain a major problem which needs to be further investigated in order to improve the biocompatibility of polymeric nanoparticles. Regarding this, many polymeric core-shell nanoparticles are being explored in various clinical phase trials, meaning that they have so far surpassed the cellular and animal toxicity requirements.

3.7. Ceramic Nanoparticles. Currently, the development of new ceramic materials for biomedical application grows hastily. Nanoscale ceramics such as hydroxyapatite (HA), 
zirconia $\left(\mathrm{ZrO}_{2}\right)$, silica $\left(\mathrm{SiO}_{2}\right)$, titanium oxide $\left(\mathrm{TiO}_{2}\right)$, and alumina $\left(\mathrm{Al}_{2} \mathrm{O}_{3}\right)$ were made from new synthetic methods to improve their physical-chemical properties seeking to reduce their cytotoxicity in biological systems. Nevertheless, the use of new ceramic materials found adverse responses by the host (in a variety of tissues, including immune system). The controlled release of drugs is one of the most exploited areas in terms of ceramic nanoparticle application in biomedicine. In this field, the dose and size are important. Also, some features that make nanoparticles a potential tool in controling drug delivery are high stability, high load capacity, easily incorporation into hydrophobic and hydrophilic systems, and different routes of administration (oral, inhalation, etc.). In addition, a variety of organic groups which may be functionalized on its surfaces allow for a directed effect [30].

3.8. Titanium Oxide Nanoparticles. The different crystalline structures of titanium dioxide make it a photocatalytic material, with broad dielectric and optical characteristics. The titanium dioxide nanoparticles have a variety of uses being the anatase stable at nanoscale, but also the most cytotoxic in a range of $3-10 \mathrm{~nm}$, which is more than 100 times in the same scale in a Rutile phase. These nanoparticles are widely used in pharmacology as drug eluting vehicles or excipient formulations. In fact, nowadays they are used in photodynamic therapy, taking advantage of their efficient photooxidation. In addition, cytotoxic aspects of nanoparticles are reduced when they are associated with other materials (e.g., hydroxyapatite) [30].

Current therapies for cancer include surgery, radio, and chemotherapy which bring undesirable effects on the human health due to unspecific target cell. Evidence indicates that therapy based on nanoparticles has potential use. With respect to this, $\mathrm{TiO}_{2}$ (titanium oxide) nanoparticles have been used in in vitro studies successfully. In fact, the $\mathrm{TiO}_{2}$ powder has been proposed as a new therapeutic agent for cancer, particularly colon cancer. Cells from human colon carcinoma were destroyed when were exposed to photoexcited nanoparticles of $\mathrm{TiO}_{2}$; also these nanoparticles have been improved with gold and platinum, thus significantly reducing the rate of survival of cancer cells. $\mathrm{TiO}_{2}$ nanoparticles are one of many efforts to improve the treatment of this disease, where the photocatalytic effect is essentially linked to the concentration of these nanoparticles. The photocatalytic activation is carried out by controlled light exposure at the tumor sites that have been treated with $\mathrm{TiO}_{2}$. The studies showed that exposure of cells from human colon carcinoma has a significant reduction in survival rate when exposed with Au-or-Pt doped $\mathrm{TiO}_{2}$ nanoparticles in comparison with the simple exposure to $\mathrm{TiO}_{2}$. These data suggests that doping of the $\mathrm{TiO}_{2}$ with $\mathrm{Au}$ and $\mathrm{Pt}$ essentially contributes to the killing of the cancer cells as compared to undoped $\mathrm{TiO}_{2}$ [31].

Sonodynamic therapy is expected to be a novel therapeutic strategy for malignant gliomas. The $\mathrm{TiO}_{2}$ nanoparticle, a photosensitizer, can be activated by ultrasound. In fact, a potential application of $\mathrm{TiO}_{2} / \mathrm{PEG}$ (polyethylene glycol) to sonodynamic therapy has been shown as a new treatment of malignant gliomas. The efficiency of sonodynamic therapy was shown using water-dispersed $\mathrm{TiO}_{2}$ nanoparticles constructed by the adsorption of chemically modified PEG on the $\mathrm{TiO}_{2}$ surface $\mathrm{TiO}_{2} / \mathrm{PEG}$. The results showed that compounds of $50 \mathrm{~nm}$ of diameter do not cross the normal blood-brain membrane, but they concentrated successfully in malignant gliomas when ultrasound methods were used [32].

On the other hand, there is clinical evidence that the exposition to different concentrations of nanoparticles including titanium oxide is phytotoxic for various animal species. Referring to humans, the introduction of nanoparticles in a wide range of industrial products promoted pregnancy complications, including spontaneous abortions. Only in the United States, it is estimated that between 1 and $3 \%$ of pregnant women had a spontaneous abortion due to concentration and distribution of these nanoparticles, and between 7 and 15\% of pregnancies has been affected by a poor fetal growth that predisposes children to cardiovascular disorders and kidney failure throughout their lives. It has been shown that exposure of rats to $\mathrm{TiO}_{2}$ nanodots produce inflammatory lesions 24 hours after exposure, particularly with nanoparticles oscillating in a size between 2 and $5 \mathrm{~nm}$. It has been found that the surface area is the most important factor associated with toxicity. Also, recent studies indicate that $\mathrm{TiO}_{2}$ nanoparticles with diameters of $35 \mathrm{~nm}$ affect the pregnancy in mice, as well as, nanoparticles injected intravenously due to accumulation in both the liver and the fetal brain. These studies were observed by bioimaging techniques after intravenous injection of fluorescence nanoparticles; images of TEM showed the presence of elements in placenta, liver, and mouse brain [33].

3.9. Silica Nanoparticles. The automatic release of potential drugs, their ease of dissolution, and ease of availability in the organism are some of the most important characteristics of the pharmaceutically active mesoporous silica molecules. However, it is difficult to determine methods to combine biocompatibility and reduce the adverse effects that these nanoparticles might exhibit in living systems, due to any slight variation in the synthesis conditions, which may result in different shapes, sizes, and subsequent physicochemical properties [30].

Moreover, there are multimodal silica nanoparticles, which are effective as markers in cancer tests and have been approved for human testing. Because of the poor selectivity in tumor tissue by markers conventionally used and the need for specificity in oncological diseases, multimodal silica nanoparticles with a diameter of $7 \mathrm{~nm}$ have been developed. Such nanoparticles are surface functionalized with arginine-glycine-aspartic acid peptide ligands and radioiodine, exhibiting a higher affinity and residence in tumors and peripheral blood fluids. Studies have shown and improved selectivity of such nanoparticles as seen by an increase in their accumulation in mouse melanoma xenografts. Such nanoparticles were approved for a first-inhuman clinical trial and were optimized for renal clearance, still showing specific tumor targeting. The silica nanoparticles were coated with a PEG layer and possess amino acid and peptide radioactive labels. The studies were conducted 
on various models of nodal metastasis in various mouse tissues and nanoparticle doses, with the results being as efficient as to begin human testing models [34]. However, these nanoparticles have shown adverse effects during the fetal development of mice, as seen for nanoparticles $70 \mathrm{~nm}$ in diameter that cross the placental membrane and cause nerve damage in the offspring. In relation to this, studies have shown embryos presenting high concentrations of silica oxide nanoparticles (up to $0.8 \mathrm{mg}$ per mouse) [33].

Recent studies in animal models have shown that inhalation of silicon dioxide nanoparticles causes pulmonary and cardiovascular disorders such as, lung inflammation, myocardial ischemia, atrial-ventricular block, and increased fibrinogen and blood viscosity. Also, DNA damage has been observed depending on the size and composition of nanoparticles which are involved on generation of free hydroxyl radicals. Furthermore, it has been found that treatment with nanoparticles of silicon dioxide, $\mathrm{SiO}_{2}$, significantly reduces cell viability by induction of apoptosis in a cell line HaCaT (human skin cells). The smaller size of these nanoparticles, the greater the rate of apoptosis (for nanoparticles 15 and $30 \mathrm{~nm}$, at a concentration of $10 \mu \mathrm{g} / \mathrm{mL}$ and $24 \mathrm{~h}$ exposure); Figure 2 presents silica nanoparticles between $10-30 \mathrm{~nm}$ in diameter. In addition, HaCaT cells provided a good model to investigate the employment of nanomaterials in cosmetic industry for skin [35].

3.10. Nanostructured Hydroxyapatite. Hydroxyapatite (HA), $\mathrm{Ca}_{10}\left(\mathrm{PO}_{4}\right)_{6}(\mathrm{OH})_{2}$, is one of the most stable forms of the calcium phosphates and the major inorganic component of bone and teeth in mammals. HA is extensively investigated, from a better understanding of the formation mechanisms in natural mineralization processes to the applicability as a biomedical or industrial material. The biocompatibility, bioactivity, bioresorbability, osteoconductivity, size dimension, morphology, and surface functionalization represent the physical and chemical properties which should be adapted in synthetic HA crystals to optimize their specific biomedical applications.

The design and synthesis of HA focus on improving its interaction with tissues. The implementation in bones and teeth is a popular choice due to mimetic bone properties. HA nanoscale crystals have demonstrated a better contact with the bone, extending the nanocrystal applications in orthopedic therapy. The nano-HA is the chosen material for surgical implantation in bone defect, and nowadays bone cements are based on calcium phosphates to initiate bone repair after surgery. The bone cement reduces metaphyseal fractures, periprosthetic, and improves the healing process of osteotomies. In addition, it can be used as a release agent for antibiotics [36].

In the dental field, nano-HA has shown excellent results in the remineralization of teeth [30]. Whereas calcium phosphate nanoparticles have shown excellent results as repair agents of dental enamel. Studies have shown that the natural tooth enamel is composed of units of HA spherical nanoparticles with diameters between 20 and $40 \mathrm{~nm}$. Experiments were carried out to determine the corrective effect

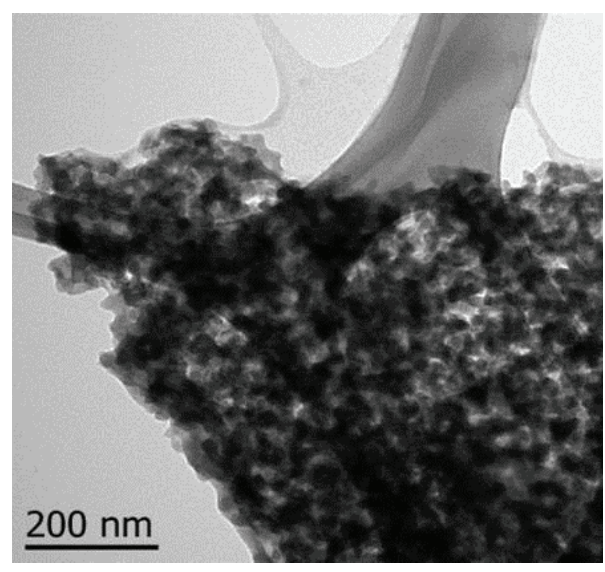

Figure 2: Transmission Electron Microscopy micrograph showing irregular-shaped of $\mathrm{SiO}_{2}$ nanoparticles with article size between 10$30 \mathrm{~nm}$.

of this nanoscale hydroxyapatite in comparison with the traditional HAP (crystals) and amorphous calcium phosphates. Samples with dental erosion of tooth enamel were used (at the first phase of caries) and treated with nanoparticles PAH. The results of scanning electron microscopy, confocal laser scanning microscopy, quantitative measurement of the adsorption, dissolution kinetics, and nanoindentation showed the strong affinity, excellent biocompatibility, mechanical improvement, and the enhancement of erosionfree by using $20 \mathrm{~nm}$ particles as the repairing agent. However, these excellent in vitro repair effects cannot be observed when conventional HAP and ACP are applied. Clearly, nano-HAP with a size of $20 \mathrm{~nm}$ shares similar characteristics to the natural building blocks of enamel so that it may be used as an effective repair material and anticaries agent. Such range of nanoparticles has therefore been suggested as an effective agent in prevention or reconstruction material in dental lesions [37].

Other authors propose the use of HAP nanoparticles as an inhibitor for human hepatoma. Hepatic carcinomas are the number one cause of death related to liver; hydroxyapatite has been shown to inhibit the proliferation of tumors. The effects of HAP nanoparticles at different doses were evaluated on the cell line BEL-7402 in vitro. On one hand, it was found that a dose of $29.30 \mathrm{mg} / \mathrm{mL}$ of nanoparticles HAPs inhibited growth, and whilst on the other hand, the cells treated with a dose between 30 and $200 \mathrm{mg} / \mathrm{mL}$ showed antiproliferative and propoptotic effect using diverse tests [38].

\section{Bioethical Issues of Nanoparticle Applications}

Nanomedicine is a relatively new area of biotechnology, where the possibilities for new therapies to treat illness and disease seem endless. Nanoparticles are already appearing in commerce as novel tools for molecular imaging, diagnosis, and drug delivery formulations. Of note, some nanoparticles have intrinsic therapeutic properties themselves. Due to such 
many existing applications, many nanotechnology products are being introduced onto the market, and yet there is still a considerable lack of knowledge of the biological effects around human exposure to such nanomaterials. Actions have already been taken to develop research strategies to evaluate the specific risks associated with exposure to each particular nanoparticle [39].

There is a significant current knowledge on the types of nanoparticles, their synthesis, and applications, as well as the associated health risks and the exposure assessment challenges facing OHS specialists. The control and prevention aspects of occupational health and safety associated with nanoparticles were also discussed in the last years [40]. In fact, knowledge in the area of hygiene pertaining to health risks is focused on integration of nanoparticle toxicity data from the literature. Nanoparticles are produced intentionally with the aim of developing new materials that exhibit certain specific properties. These properties are related to at least one of their dimensions, which must be less than 100 nanometers $(\mathrm{nm})$. Recent studies of the biological effects of nanoparticles show signs that some manufactured nanoparticles display unexpected toxicity to living organisms. Some of these particles can become potentially harmful and even cause deleterious human health effects [41].

The toxicological evaluation of the new chemical compounds is an area that in this century has been relevant. There is almost no other scientific field in which the core experimental protocols have remained nearly unchanged for more than 40 years. Yet consumers continually increase their expectations about the safety of products. One recent effect of this was the instigation of the largest safety assessment of chemicals that has ever been carried out: the European Union introduced the regulation known as Registration, Evaluation, Authorisation and Restriction of Chemicals (REACH) by legislation in 2007. Whereas new chemicals have been systematically evaluated in the European Union and the United States for about a quarter of a century, the safety of any chemicals produced before 1981 (which includes $97 \%$ of the major chemicals in use and more than $99 \%$ of chemicals produced by volume) has not necessarily been properly addressed [42].

As chemical substances, nanoparticles may first come under the regulations for chemical agents. Furthermore, when the substances are integrated into a manufactured product, they are likely to be subject to diverse regulations concerning the marketing of products containing them. They would then be covered, for example, by the various directives on biocides, cosmetics products, dental materials, and drugs for human and veterinary use. The manufactured nanoparticle would then be assessed as part of the product as used by a professional or consumer. Current law includes no texts applicable to manufactured nanoparticles as such. There have been three points proposed: (i) a complete series of existing regulations certainly appear potentially applicable, (ii) because none targets them specifically; however, their implementation is very uncertain, and (iii) accordingly existing laws must be clarified and new measures adopted swiftly.

\section{Concluding Remarks and Future Considerations}

The application of nanotechnology to drug delivery has already had a significant impact on many areas of medicine. Currently, more than 20 nanoparticle therapeutics are in clinical use, validating the ability of nanoparticles to improve the therapeutic index of drugs. In addition to the already approved nanoparticles, numerous other nanoparticle platforms are currently under various stages of preclinical and clinical development, including various liposomes, polymeric micelles, dendrimers, quantum dots, gold nanoparticles, and ceramic nanoparticles. More complex systems such as multifunctional nanoparticles that are concurrently capable of targeting, imaging, and therapy are subject of future research.

However, we should be aware of possible unwanted sideeffects. Nanotechnology means new materials and components, which can be included in many different existing products, or enable new products. Despite potential benefits of nanotechnology, there are potential ethical issues, which need desirable solutions.

The currently approved nanoparticle systems have in some cases improved the therapeutic index of drugs by reducing drug toxicity or enhancing drug efficacy. Future research efforts need to be directed towards finding new methods for nanotoxicology, recognition of biological effects of nanoparticles in the environment, and creation of the bases of nanobiomonitoring.

\section{Conflict of Interests}

The authors declared that they have no conflict of interests.

\section{Acknowledgments}

The authors wish to thank for technical support: Dra. Genoveva Hernández-Padrón, Mtra. Ma. Lourdes Palma Tirado, Dra. Marina Vega, and Miguel A. Arellano.

\section{References}

[1] L. Yildirimer, N. T. K. Thanh, M. Loizidou, and A. M. Seifalian, "Toxicological considerations of clinically applicable nanoparticles," Nano Today, vol. 6, no. 6, pp. 585-607, 2011.

[2] G. Lövestam, H. Rauscher, G. Roebben, B.S. Klüttgen et al., "Considerations on a definition of nanomaterial for regulatory purposes," JRC Reference Reports JRC58726, Publications Office of the European Union, 2010.

[3] J. A. Kim, Aberg C., A. Salvati, and K. A. Dawson, "Role of cell cycle on the cellular uptake and dilution of nanoparticles in a cell population," Nature Nanotechnology, vol. 7, no. 1, pp. 62-68, 2012.

[4] E. Cairo, "Nanotechnology based drug delivery in mucosal immune diseases: hype or hope?" Mucosal Immunology, vol. 5, pp. 2-3, 2012.

[5] J. V. Georgieva, D. Kalicharan, P. O. Couraud et al., "Surface characteristics of nanoparticles determine their intracellular 
fate in and processing by human blood-brain barrier endothelial cells in vitro," Molecular Therapy, vol. 19, no. 2, pp. 318 325, 2011.

[6] P. H. M. Hoet, A. Nemmar, and B. Nemery, "Health impact of nanomaterials?” Nature Biotechnology, vol. 22, no. 1, p. 19, 2004.

[7] I. Linkov, M. E. Bates, L. J. Canis, T. P. Seager, and J. M. Keisler, "A decision-directed approach for prioritizing research into the impact of nanomaterials on the environment and human health," Nature Nanotechnology, vol. 6, no. 12, pp. 784-787, 2011.

[8] K. K. Jain, The Handbook of Nanomedicine, Humana/Springer, Totowa, NJ, USA, 2008.

[9] K. Cho, X. Wang, S. Nie, Z. Chen, and D. M. Shin, "Therapeutic nanoparticles for drug delivery in cancer," Clinical Cancer Research, vol. 14, no. 5, pp. 1310-1316, 2008.

[10] W. J. Gradishar, S. Tjulandin, N. Davidson et al., "Phase III trial of nanoparticle albumin-bound paclitaxel compared with polyethylated castor oil-based paclitaxel in women with breast cancer," Journal of Clinical Oncology, vol. 23, no. 31, pp. 77947803, 2005.

[11] A. Kumari, S. K. Yadav, and S. C. Yadav, "Biodegradable polymeric nanoparticles based drug delivery systems," Colloids and Surfaces B, vol. 75, no. 1, pp. 1-18, 2010.

[12] R. C. Mundargi, V. R. Babu, V. Rangaswamy, P. Patel, and T. M. Aminabhavi, "Nano/micro technologies for delivering macromolecular therapeutics using poly(d,l-lactide-coglycolide) and its derivatives," Journal of Controlled Release, vol. 125, no. 3, pp. 193-209, 2008.

[13] Z. S. Haidar, M. Tabrizian, and R. C. Hamdy, "A hybrid rhOP-1 delivery system enhances new bone regeneration and consolidation in a rabbit model of distraction osteogenesis," Growth Factors, vol. 28, no. 1, pp. 44-55, 2010.

[14] M. H. Dufresne, D. Le Garrec, V. Sant, J. C. Leroux, and M. Ranger, "Preparation and characterization of water-soluble $\mathrm{pH}$-sensitive nanocarriers for drug delivery," International Journal of Pharmaceutics, vol. 277, no. 1-2, pp. 81-90, 2004.

[15] V. A. Sethuraman and Y. H. Bae, "TAT peptide-based micelle system for potential active targeting of anti-cancer agents to acidic solid tumors," Journal of Controlled Release, vol. 118, no. 2, pp. 216-224, 2007.

[16] H. Otsuka, Y. Nagasaki, and K. Kataoka, "PEGylated nanoparticles for biological and pharmaceutical applications," Advanced Drug Delivery Reviews, vol. 55, no. 3, pp. 403-419, 2003.

[17] N. Welsch, A. Wittemann, and M. Ballauff, "Enhance activity of enzymes immobilized in thermoresponsive core-shell microgels," Journal of Physical Chemistry B, vol. 113, no. 49, pp. 16039-16045, 2009.

[18] K. S. Soppimath, T. M. Aminabhavi, A. R. Kulkarni, and W. E. Rudzinski, "Biodegradable polymeric nanoparticles as drug delivery devices," Journal of Controlled Release, vol. 70, no. 1-2, pp. 1-20, 2001.

[19] C. Roney, P. Kulkarni, V. Arora et al., "Targeted nanoparticles for drug delivery through the blood-brain barrier for Alzheimer's disease," Journal of Controlled Release, vol. 108, no. 2-3, pp. 193-214, 2005.

[20] P. V. Kulkarni, C. A. Roney, P. P. Antich, F. J. Bonte, A. V. Raghu, and T. M. Aminabhavi, "Quinoline-n-butylcyanoacrylate-based nanoparticles for brain targeting for the diagnosis of Alzheimer's disease," Wiley Interdisciplinary Reviews: Nanomedicine and Nanobiotechnology, vol. 2, no. 1, pp. 35-47, 2010.
[21] M. Doshi, "Dendrimer and its application," International Journal of Pharmaceutical Sciences Review and Research, vol. 7, no. 2, pp. 104-111, 2011.

[22] R. K. Tekade, P. V. Kumar, and N. K. Jain, "Dendrimers in oncology: an expanding horizon," Chemical Reviews, vol. 109, no. 1, pp. 49-87, 2009.

[23] S. A. Agnihotri, N. N. Mallikarjuna, and T. M. Aminabhavi, "Recent advances on chitosan-based micro- and nanoparticles in drug delivery," Journal of Controlled Release, vol. 100, no. 1, pp. 5-28, 2004.

[24] W. E. Rudzinski and T. M. Aminabhavi, "Chitosan as a carrier for targeted delivery of small interfering RNA," Journal of Controlled Release, vol. 125, pp. 193-209, 2008.

[25] K. Chaturvedi, K. Ganguly, A. R. Kulkarni et al., "Cyclodextrin-based siRNA delivery nanocarriers: a state-of-the-art review," Expert Opinion on Drug Delivery, vol. 8, no. 11, pp. 1455-1468, 2011.

[26] V. R. Babu, P. Patel, R. C. Mundargi, V. Rangaswamy, and T. M. Aminabhavi, "Developments in polymeric devices for oral insulin delivery," Expert Opinion on Drug Delivery, vol. 5, no. 4, pp. 403-415, 2008.

[27] O. V. Salata, "Applications of nanoparticles in biology and medicine," Journal of Nanobiotechnology, vol. 2, article 3, 2004.

[28] C. Damgé, P. Maincent, and N. Ubrich, "Oral delivery of insulin associated to polymeric nanoparticles in diabetic rats," Journal of Controlled Release, vol. 117, no. 2, pp. 163-170, 2007.

[29] J. C. Pickup, Z. L. Zhi, F. Khan, T. Saxl, and D. J. S. Birch, "Nanomedicine and its potential in diabetes research and practice," Diabetes/Metabolism Research and Reviews, vol. 24, no. 8, pp. 604-610, 2008.

[30] B. Fadeel and A. E. García-Bennett, "Better safe than sorry: understanding the toxicological properties of inorganic nanoparticles manufactured for biomedical applications," Advanced Drug Delivery Reviews, vol. 62, no. 3, pp. 362-374, 2010.

[31] C. Lazau, L. Mocanu, I. Miron, P. Sfirloaga et al., "Consideration regarding the use of $\mathrm{TiO}_{2}$ doped nanoparticles in medicine," Digest Journal of Nanomaterials and Biostructures, vol. 2, pp. 257-263, 2007.

[32] S. Yamaguchi, H. Kobayashi, T. Narita et al., "Sonodynamic therapy using water-dispersed $\mathrm{TiO}_{2}$-polyethylene glycol compound on glioma cells: comparison of cytotoxic mechanism with photodynamic therapy," Ultrasonics Sonochemistry, vol. 18, no. 5, pp. 1197-1204, 2011.

[33] K. Yamashita, Y. Yoshioka, K. Higashisaka et al., "Silica and titanium dioxide nanoparticles cause pregnancy complications in mice," Nature Nanotechnology, vol. 6, no. 5, pp. 321328, 2011.

[34] M. Benezra, O. Penate-Medina, P. B. Zanzonico et al., "Multimodal silica nanoparticles are effective cancer-targeted probes in a model of human melanoma," Journal of Clinical Investigation, vol. 121, no. 7, pp. 2768-2780, 2011.

[35] X. Yang, J. Liu, H. He et al., " $\mathrm{SiO}_{2}$ nanoparticles induce cytotoxicity and protein expression alteration in HaCaT cells," Particle and Fibre Toxicology, vol. 7, article 1, 2010.

[36] N. Roveri and M. Iafisco, "Evolving application of biomimetic nanostructured hydroxyapatite," Nanotechnology, Science and Applications, vol. 3, no. 1, pp. 107-125, 2010.

[37] L. Li, H. Pan, J. Tao et al., "Repair of enamel by using hydroxyapatite nanoparticles as the building blocks," Journal of Materials Chemistry, vol. 18, no. 34, pp. 4079-4084, 2008. 
[38] Z. S. Liu, S. L. Tang, and Z. L. Ai, "Effects of hydroxyapatite nanoparticles on proliferation and apoptosis of human hepatoma BEL-7402 cells," World Journal of Gastroenterology, vol. 9, no. 9, pp. 1968-1971, 2003.

[39] N. Sanvicens and M. P. Marco, "Multifunctional nanoparticles: properties and prospects for their use in human medicine," Trends in Biotechnology, vol. 26, no. 8, pp. 425-433, 2008.

[40] C. Ostiguy, G. Lapointe, M. Trottier, L. Ménard L et al., "The health effects of nanoparticles," IRSST Report R-451, 2006.

[41] C. Ostiguy, B. Soucy, C. Woods, L. Ménard, and M. Trottier, "Chemical substances and biological agents: studies and research projects health, effects of nanoparticles second edition," IRSST Report R-589, Montréal, Canada, 2008.

[42] T. Hartung, "Toxicology for the twenty-first century," Nature, vol. 460, no. 7252, pp. 208-212, 2009. 

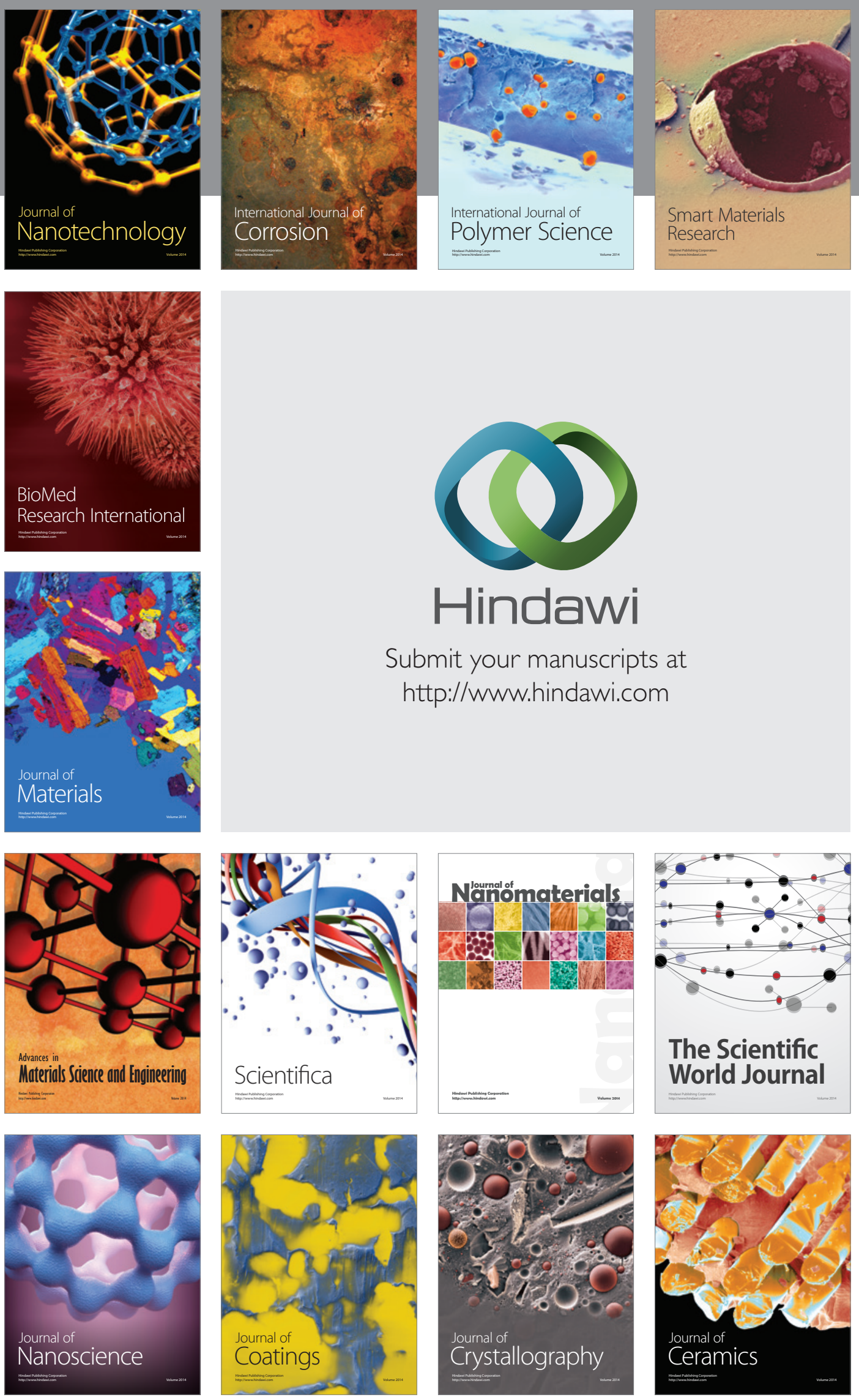

The Scientific World Journal

Submit your manuscripts at

http://www.hindawi.com

\section{World Journal}

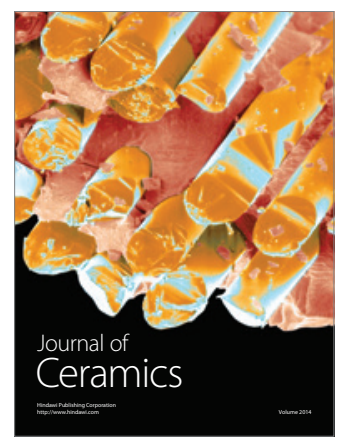

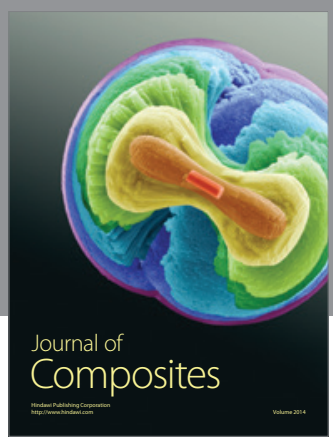
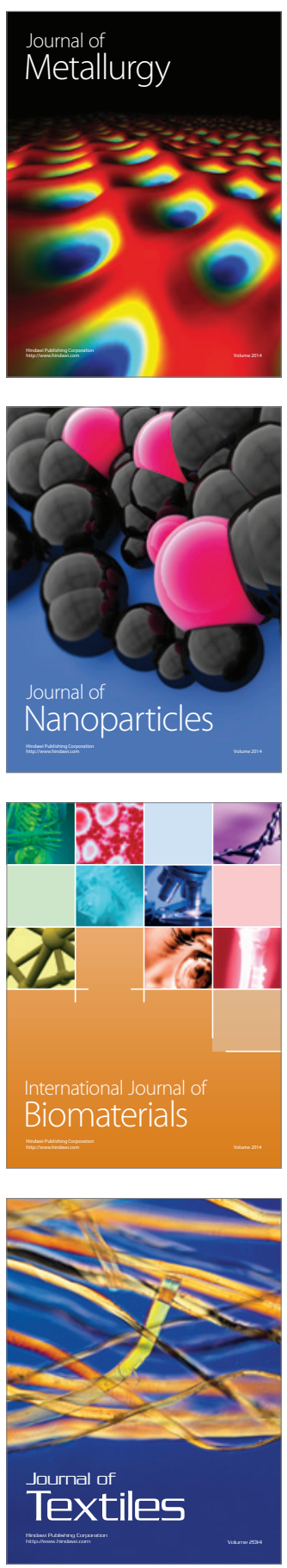